\title{
Nanostructured titanium dioxide coatings prepared by Aerosol Assisted Chemical Vapour Deposition (AACVD)
}

\author{
Megan Taylor ${ }^{\mathrm{a}}$, Robert C. Pullar ${ }^{\mathrm{b}}$, Ivan P. Parkin ${ }^{\mathrm{a}}$, Clara Piccirillo ${ }^{\mathrm{c}, *}$ \\ ${ }^{\text {a }}$ Chemistry Department, University College London, London, United Kingdom \\ ${ }^{\mathrm{b}}$ Department of Materials and Ceramic Engineering, CICECO - Aveiro Institute of Materials, University of Aveiro, Aveiro, Portugal \\ ${ }^{\mathrm{c}}$ CNR NANOTEC, Institute of Nanotechnology, Lecce, Italy
}

\section{A R T I C L E I N F O}

\section{Keywords:}

Chemical vapour deposition

Titanium dioxide

Acetyl acetone

Nanostructure

Photocatalysis

\begin{abstract}
A B S T R A C T
Titanium dioxide is a compound of great interest, due to its functional properties; one of its most important uses is as a photocatalyst. $\mathrm{TiO}_{2}$ coatings can be deposited using different techniques. Aerosol Assisted Chemical Vapour Deposition (AACVD) is particularly interesting, as high temperature or pressure are not necessary to generate the gaseous precursors. Furthermore, by carefully choosing the deposition conditions (i.e. deposition temperature, solvent), it is possible to obtain deposits with different morphology and, consequently, different functional properties. In this paper we present the synthesis of titanium dioxide coatings with AACVD using complexes between titanium isopropoxide (TIPP) and acetyl acetone (acac) as precursors. Deposition experiments were performed using different ratios of TIPP to acac, to assess the effect on the composition of the coatings, their morphology and photocatalytic activity. Results showed that the use of acac led to nanostructured titanium dioxide (nanoparticles of about 10-25 nm diameter). Raman analysis showed the presence of both anatase and rutile phases. XPS analysis indicated the presence of residual carbonaceous species in the coatings; despite this, they displayed photocatalytic properties similar or superior to AACVD films without carbon. Photocatalytic tests, performed measuring the Formal Quantum Efficiency (FQE) and the Formal Quantum Yield (FQY) in the degradation of resazurin, showed that a acac:TIPP ratio equal to 1 led to the material with the highest performance, as the FQE value was about three times higher than that for the coating prepared with TIPP alone. Overall the complexes between TIPP and acac are promising precursors for the AACVD technique, leading to nanostructured coatings with enhanced performance.
\end{abstract}

\section{Introduction}

Titanium dioxide $\left(\mathrm{TiO}_{2}\right)$ is a material which has attracted ever growing interest, due to its many useful properties. $\mathrm{TiO}_{2}$ can exist in three common forms, namely anatase, rutile and brookite. It is resistant to corrosion in different environments, and non-toxic [1]. $\mathrm{TiO}_{2}$ is the most used and effective photocatalytic material - in particular, the anatase form shows very high photocatalytic efficiency, with a band gap of $3.2 \mathrm{eV}$ [2]. The co-presence of different phases, in particular rutile, can lead to an enhancement in the photocatalytic performance [3].

The mechanism of the photocatalytic activity is well known: under light irradiation (energy equal or higher than the value of the band gap), a promotion of an electron from the valence band to the conduction band takes place, leading to the creation of a positively-charged hole. Both the electron and the hole (charged reactive species) can successively react with chemical compound(s) they are in contact with. In case of organic and/or gaseous molecules, for instance, this can lead to their degradation [4]. In the case of microorganisms, on the other hand, such charged species can cause their death or deactivation [5]. One of the key factors for the material to be an effective photocatalyst is the extent of the recombination of the charges (electrons and holes), which inactivates them. Such recombination can be affected by several parameters, including morphology, crystallinity and possible preferred orientation $[6,7]$.

To prepare titanium dioxide, the most common precursor generally employed is titanium isopropoxide ( $\mathrm{Ti}\left(\mathrm{OCH}\left(\mathrm{CH}_{3}\right)_{2}\right)_{4}$, TIPP); the main problem associated with its use, however, is its stability. TIPP tends to hydrolyse when in contact with moisture, which may lead to the formation of titanium dioxide crystals without a proper control of their shape and size [8]. Acetyl acetone (a di-ketone with formula $\mathrm{C}_{5} \mathrm{H}_{8} \mathrm{O}_{2}$, acac) has been reported to stabilise the titanium (IV) ion in solution by 
forming an acetyl acetonate complex [9]; indeed, acac-stabilised TIPP was employed to prepare $\mathrm{TiO}_{2}$ both in form of crystals/powder and thin films $[10,11]$. Different ratios between the acac and titanium have been studied, from $<1$ to $4[10,12,13]$.

Titanium dioxide coatings have been prepared using different methodologies [11,14,15]; between them, Chemical Vapour Deposition (CVD) was shown to be very suitable to obtain $\mathrm{TiO}_{2}$ with tailored morphology [16-18] and doped with different elements [19,20]. Aerosol Assisted CVD (AACVD), in particular, was successfully used to deposit the different phases of $\mathrm{TiO}_{2}$ [21], as well as doped $\mathrm{TiO}_{2}$ coatings [22]. Despite the interesting results achieved with AACVD, however, titania coatings prepared with this methodology generally did not show a nanostructured morphology; indeed, deposits with a needle-like structure or spherical $100-200 \mathrm{~nm}$ crystallites were prepared [21], while only one study of nanostructured coating was reported [23]. Moreover, while several studies have been performed to investigate the effect of different solvents, as well as deposition substrates [21,23,24], no investigation has been performed into the effect that a different deposition precursor may have on the characteristics and performance of the films, and TIPP alone was always employed.

In the present work, we report for the first time the synthesis of titanium dioxide with AACVD using TIPP modified with acac as a precursor. Different ratios between TIPP and the acac ligand were tested, to assess which were the best deposition conditions. Samples were characterised by different analytical techniques (XRD, Raman, SEM, XPS), to determine the effect of the precursor on the composition of the coatings and their morphology; their functional properties (i.e. photocatalytic activity) were also assessed. Results showed that by choosing the appropriate ratio between the precursor and the ligand, films with enhanced photocatalytic activity could be prepared.

\section{Materials and methods}

Titanium isopropoxide, acetyl acetone and dry toluene were all purchased from Aldrich; they were used without further treatment or purification. The complex formed between Ti(IV) and acac was studied by UV spectroscopy. To do this, different amounts of acac were dissolved in dry toluene (total volume $10 \mathrm{~mL}$ ); TIPP $(0.5 \mathrm{~mL})$ was successively added to the solution, which was gently stirred for about 10 min. After this time, the solution was analysed with a Varian Cary300SCAN spectrometer; when necessary, appropriate dilution was performed with toluene, to avoid the saturation of the signal. Different molar ratios between the ligand and the metal from 0.5 to 4 were tested.

Titanium dioxide coatings were deposited using a cold-wall horizontal reactor, previously described [25].

For the depositions, TIPP-acac solutions prepared as described above were employed. An aerosol was generated from each solution using an Asiamist 1308 ultrasonic humidifier; such aerosol was carried to the deposition chamber using inert $\mathrm{N}_{2}$ with a flow rate of $1.5 \mathrm{~L} / \mathrm{min}$. Float glass sheets (Pilkington NSG TEC ${ }^{\mathrm{TM}} \mathrm{SB}$, full description at https:// www.pilkington.com/en/global/products/product-categories/specialapplications/nsg-tec-for-technical-applications\#overview) were employed as deposition substrate; the glass had a $50 \mathrm{~nm}$ thick $\mathrm{SiO}_{2}$ layer to suppress the diffusion of the ions from the glass; all depositions were performed at $450{ }^{\circ} \mathrm{C}$. To set and control the temperature of the substrate, a Tempatron model 4800 thermostat - equipped with a Pt-Rh thermocouple - was used. The deposition was continued until the whole solution was used (about $45 \mathrm{~min}$ ). Subsequently, the system was cooled to room temperature under a $\mathrm{N}_{2}$ flow.

X-ray diffraction (XRD) patterns were acquired with a Bruker D8 diffractometer, using filtered radiation in the reflection mode, with a glancing angle incidence of $1.5^{\circ}$. Raman spectroscopy was performed using a Horiba Jobin Yvon HR800 with a $532 \mathrm{~nm}$ laser. To determine the morphology of the coatings, Scanning Electron Microscopy (SEM) analysis was performed with a Hitachi S-4100, at $15 \mathrm{kV}$. Before the analysis, the coatings were sputtered with gold, to avoid charge accumulation. X-ray photoelectron spectroscopy (XPS) spectra were acquired using Thermo Scientific K-alpha photoelectron equipment, with monochromatic Al-ka radiation. High resolution scans were acquired for the peaks of Ti(2p), $\mathrm{O}(1 \mathrm{~s})$ and $\mathrm{C}(1 \mathrm{~s})$ at a passing energy of $50 \mathrm{eV}$. Data were analysed using CasaXPS software, which was calibrated to the adventitious carbon at $284.5 \mathrm{eV}$. Atomic Force Microcopy (AFM) was performed using a XE-100 microscope (PSIA, Korea); images were acquired at room temperature in non-contact mode. UV spectra of the coatings were acquired using a Perkin Elmer Lambda 1050, with a 150 $\mathrm{mm}$ InGaAs integrating sphere. The measurements were taken in reflection mode (some samples were too dark to use the transmittance mode); data were converted into the Kubelka-Munk function using the equation:

$k=\frac{(1-R)^{2}}{2 R}$

The photocatalytic activity of the deposits was measured using a resazurin based "intelligent ink", prepared as previously described [26]. The surfaces of the coatings (about $0.8 \mathrm{~cm}^{2}$ ) were evenly covered with the dye. Photocatalytic reduction of the dye by UVA light (ViberLourmat lamp, $\lambda=365 \mathrm{~nm}$, radiation energy $1.2 \mathrm{~mW} / \mathrm{cm}^{2}$ ) was measured by UV-vis spectroscopy (Perkin Elmer Lambda 950). The formal quantum efficiency (FQE) was calculated by dividing the rate of dye molecules degraded by the photon flux. The formal quantum yield (FQY) was calculated by dividing the rate of the degradation of the dye molecule by the number of photons absorbed. Both photon flux and photon absorption were measured with a UVX radiometer, with a detector for $365 \mathrm{~nm}$ radiation attached.

\section{Results and discussion}

\subsection{Analysis of the precursor}

Prior to the depositions, the complex formed between Ti(IV) and acac in different ratios was studied; the corresponding UV-vis spectra are shown in Fig. 1, and a photo of the solutions is shown in the SI (Fig. SI1). It can be seen that, when no acac is added to the solution, there is no absorbance in the visible (i.e. the solution is colourless), the spectrum showing just one broad peak with a maximum for $\lambda$ around 320 $\mathrm{nm}$. Adding acac, the solutions become coloured (from yellow to pale brown, see Fig. SI1), in agreement with literature [13], and different peaks can be observed in the spectra, while the $320 \mathrm{~nm}$ signal almost

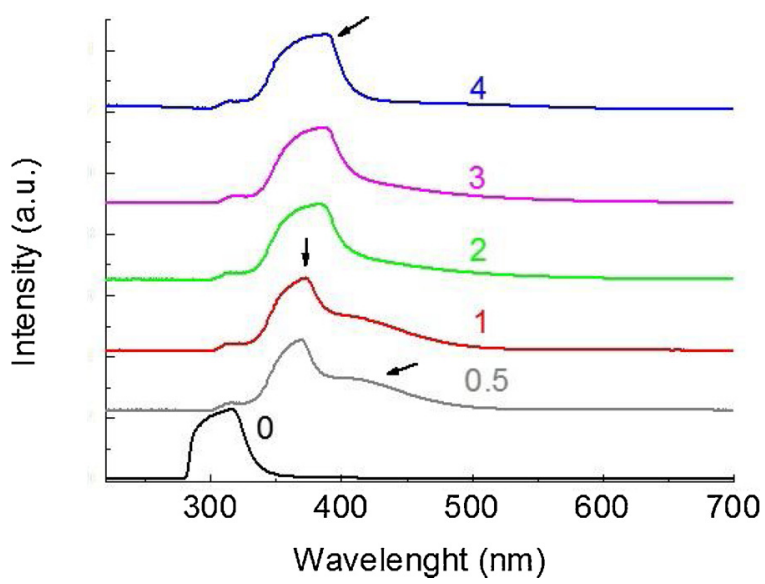

Fig. 1. UV spectra of the complexes between TIPP and acac in different proportions; the number close to each curve indicates the ratio of the acac complexing agent to the TIPP.

Note: the curves were shifted on the Y axis to separate them and make them easier to see. Arrows indicate the shoulder present at 0.5 and 1, and the shift of the peak from 370 to $390 \mathrm{~nm}$. 
disappears. For lower acac-Ti(IV) ratios (0.5 and 1), a strong peak is detected at about $370 \mathrm{~nm}$; moreover, a shoulder at about $415 \mathrm{~nm}$ is also observed. For higher acac concentrations, this shoulder peak disappears, as at the same time the stronger peak shifts to a slightly higher wavelength of about $390 \mathrm{~nm}$. As can be seen from the picture, the solutions are all transparent, with no colloidal formation, confirming the stability of the complex.

According to literature, the formation of the complex between $\mathrm{Ti}$ (IV) and acac takes place by substitution, i.e. an acac group replaces the isopropoxide bonded to Ti(IV) [27]; it is likely, however, that the structure and coordination of the complex changes, as acac is a bidentade ligand [28]. Indeed, the use of $\beta$-diketonate molecules is known to lead to a higher Ti (IV) coordination number [29]. Considering the spectra of Fig. 1, it seems that the addition of smaller quantities of acac leads to the formation of a complex with mixed geometry, i.e. with more than one absorption peak. For higher acac concentrations (acac-Ti equal to 2 , or greater), on the other hand, only a single peak at a slightly higher wavelength was observed, suggesting a single geometry. With acac-Ti ratios up to 4 no further changes were observed in the spectra, implying no significant differences in the geometry of the complex.

Several investigations were published which studied in detail the geometry of Ti(IV) complexes with different ligands, including acac $[30,31]$. Literature reports that the special arrangement can be affected by several parameters, such as the nature of the solvent. Moreover, equilibrium between different forms can be reached over time [28]. A deeper study of the Ti(IV)-acac complex is beyond the scope of this work, whose main focus is the use of such complexes as precursors for AACVD deposition.

\subsection{Deposition experiments}

Based on these results, AACVD experiments were performed usng the acac concentrations, and acac:Ti ratios of $0.5,1$ and 4, shown in Table 1. A deposition was also performed with no acac, for the sample to be used as reference.

All deposits showed good adhesion and passed the Scotch tape test; there were, however, differences in the appearance of the samples according to the acac content. Coatings prepared with the highest ligand content in solution (acac:Ti ratio of 4) produced a completely covered glass substrate, while for the other depositions conditions only about three quarters of the glass was coated. As already mentioned above, the complexes between $\mathrm{Ti}(\mathrm{IV})$ and acac are generally more stable / resistant to hydrolysis than with iso-propoxide, indicating that more energy may be necessary for the acac complex to be degraded, and for the titanium to react on the surface of the glass. This could lead to the molecule being in contact for longer periods with the heated substrate without reacting, i.e. arriving at the far end of the deposition substrate, at the opposite end from the reactor inlet.

It was also observed that using higher acac:Ti ratios, the deposits possessed a darker appearance. This feature can be explained considering that the acac ligand $\left(\mathrm{C}_{5} \mathrm{H}_{8} \mathrm{O}_{2}\right)$ contains more carbon than the isopropoxide group $\left(\mathrm{C}_{3} \mathrm{H}_{7} \mathrm{O}^{-}\right)$; as the depositions were performed at $450{ }^{\circ} \mathrm{C}$, the combustion of the organic matter is not complete at this temperature, hence leaving some carbonaceous material. The higher acac complex stability may have also played a role.

Table 1

List of the prepared samples.

\begin{tabular}{ll}
\hline Sample name & acac:Ti ratio \\
\hline AA_0 & 0 \\
AA_0.5 & 0.5 \\
AA_1 & 1 \\
AA_4 & 4 \\
\hline
\end{tabular}

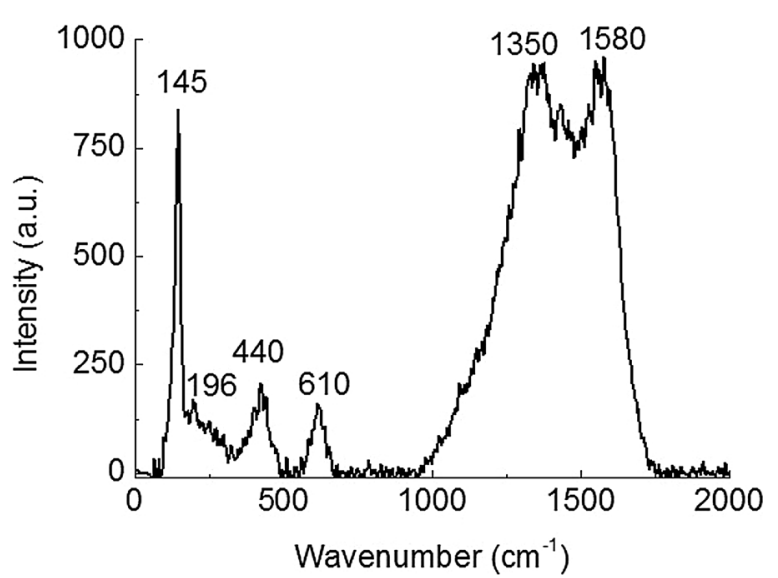

Fig. 2. Raman spectrum of sample AA_1.

XRD patterns of the different samples were acquired to determine their phase composition, and data are shown in Fig. SI2. It can be seen that sample AA_0, prepared with no acac, shows the characteristic anatase peak at $2 \theta=25^{\circ}$. When acac was used, however, no peak could be detected, as can be seen in Fig. SI2 for sample AA_0.5; the patterns for the other samples showed similar features. This indicates that they are not crystalline, but amorphous.

Alternatively, Raman measurements were performed; Fig. 2 shows as an example the spectrum for sample AA_1.

It can be seen that a sharp peak is present at $145 \mathrm{~cm}^{-1}$, which corresponds to anatase $\mathrm{TiO}_{2}$ [7], and a much smaller peak at $196 \mathrm{~cm}^{-1}$ also belongs to the same phase. Additional peaks at 440 and $610 \mathrm{~cm}^{-1}$ are also present, which are associated with rutile $\mathrm{TiO}_{2}$ [32]. Samples AA_0.5 and AA_4, deposited using acac, showed similar features, i.e. the presence of rutile. Previous work, on the other hand, showed no rutile formation was observed using just TIPP for AACVD, with no acac [25], with only anatase being observed. This indicates that the acac presence has a significant effect on the deposition mechanisms, since a second $\mathrm{TiO}_{2}$ phase is formed, even when the lowest amount of acac was used. Rutile formation is generally observed in samples prepared or treated at temperatures from $500{ }^{\circ} \mathrm{C}$ onwards [33,34], although a mixed phase material containing both anatase and rutile in sizeable quantities was observed in $\mathrm{TiO}_{2}$ nanopowders produced by sol-gel and heated at $450{ }^{\circ} \mathrm{C}$ [4]. As Raman is not a quantitative technique, it is not possible to quantify the proportion of each phase. However, the rutile peaks are well defined, indicating a significant presence well above the detection limit. Other peaks that can be observed in the spectrum correspond to residual carbon contamination - the D and $\mathrm{G}$ bands of graphite-like materials, at 1350 and $1580 \mathrm{~cm}^{-1}$, respectively. These peaks were not detected for $\mathrm{TiO}_{2}$ deposited without acac.

Fig. 3 shows the SEM images for the deposited coatings; it can be seen that the addition of the acac to the solution deposition significantly affects also the morphology of the films. When no acac was used (sample AA_0, Fig. 3(a)), in fact, the film showed the same features already observed before [21,25], i.e. crystalline needles with the dimensions of about $400 \mathrm{~nm}$ in length and less than $100 \mathrm{~nm}$ in thickness, with nucleation centres present on the sides. Such elongated tetragonal needles are typical of the anatase titania crystalline phase. In the presence of acac, on the other hand, the titania coatings exhibit a nanometric morphology. For both samples AA_0.5 and AA_1 (Fig. 3(b) and (c) respectively), rounded nanoparticles with dimensions of between $15-25 \mathrm{~nm}$, and $10-20 \mathrm{~nm}$, can be observed respectively. Although the particle size decreases slightly with increasing acac, the size distribution of the nanoparticles is quite uniform in both cases. With higher acac content (sample AA_4, Fig. 3(d)), a similar morphology can be observed, with a size range of $10-20 \mathrm{~nm}$, although the average diameter is slightly smaller, and more variation in the size distribution can be observed, with a greater number of smaller nanoparticles. 

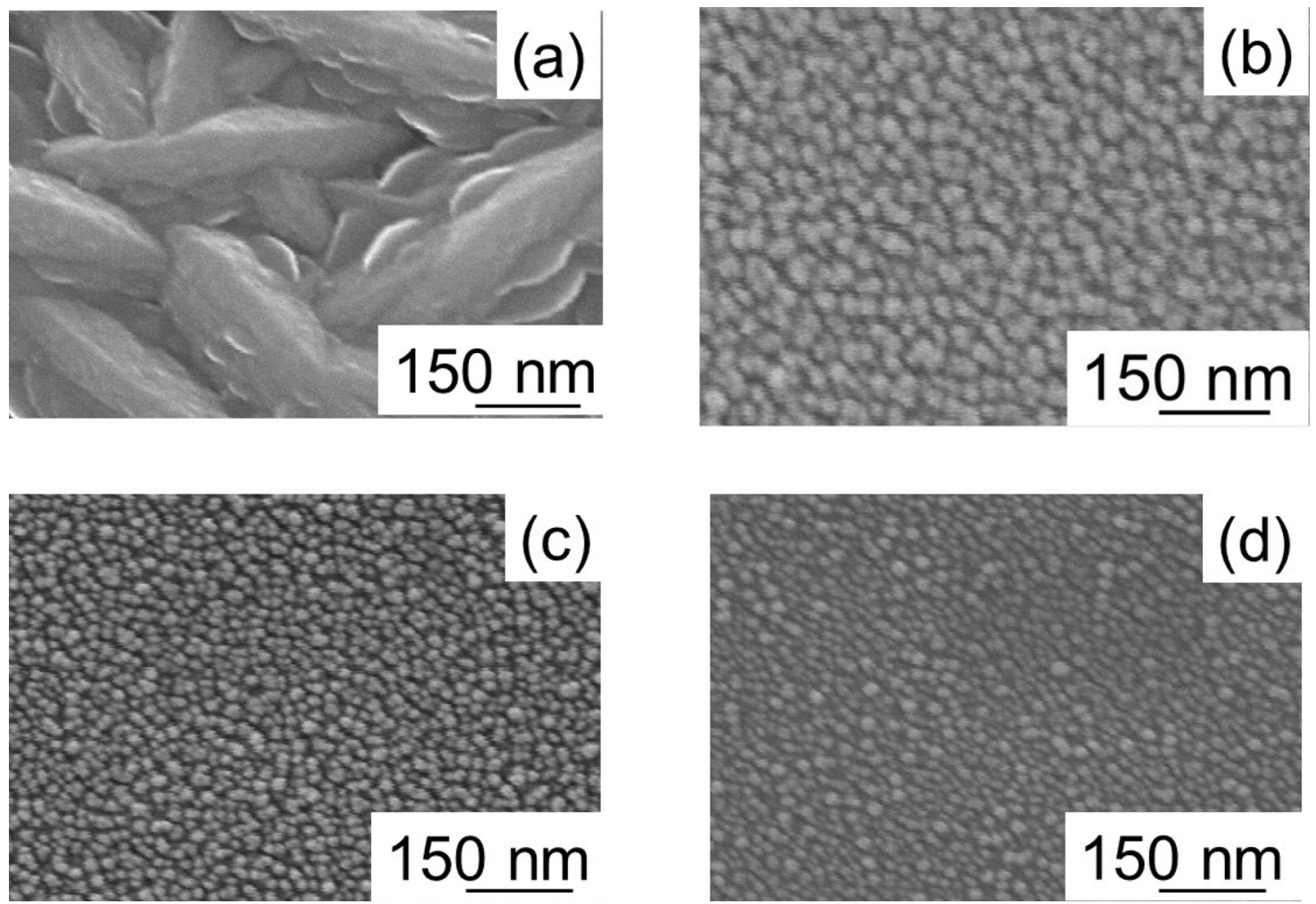

Fig. 3. SEM micrographs for the coatings deposited in different conditions. (a) AA_0, (b) AA_0.5, (c) AA_1, (d) AA_4.
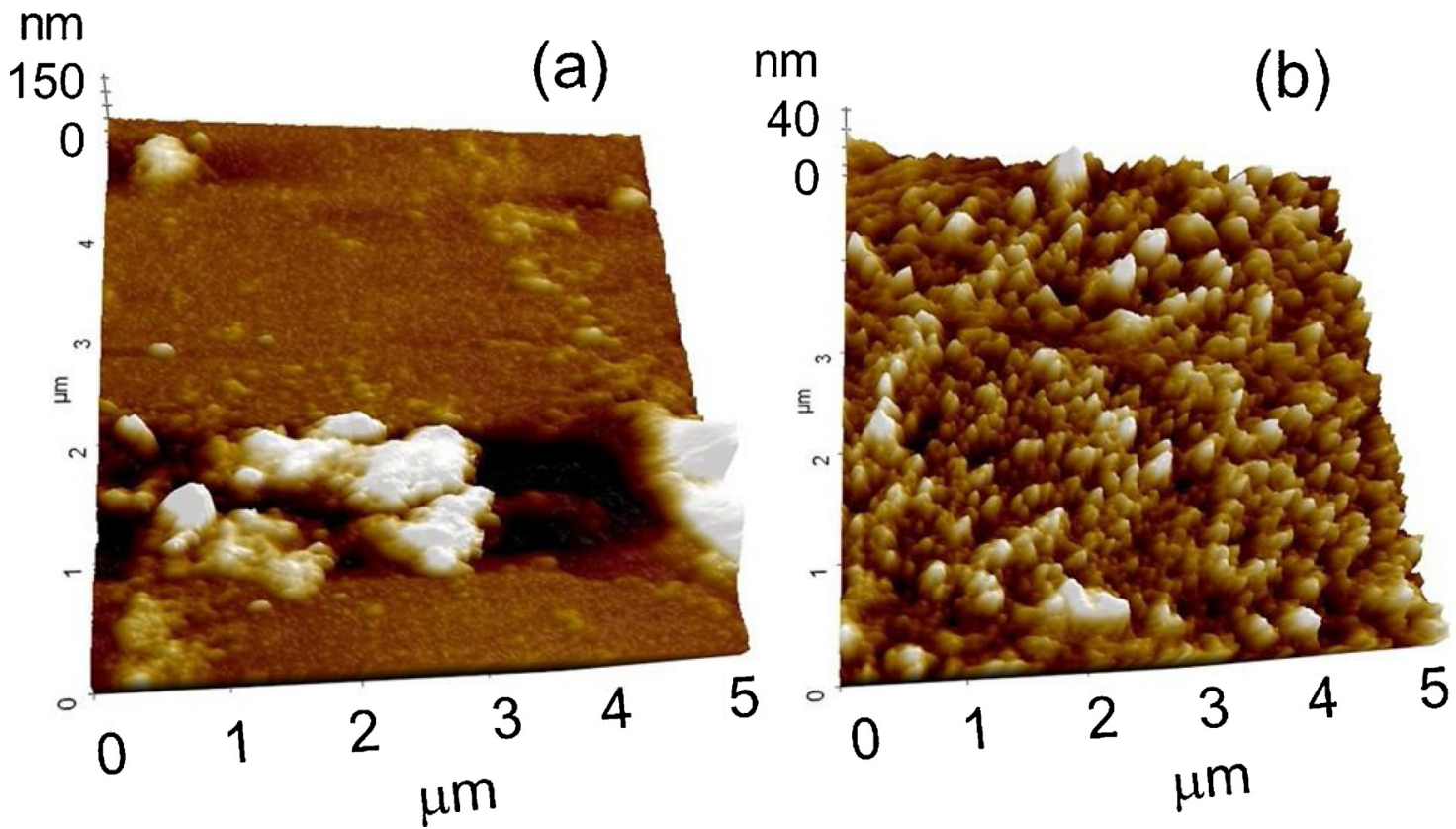

Fig. 4. AFM images for samples (a) AA_0 and (b) AA_4.

A cross section micrograph of sample AA 0.5 is shown in Fig. SI3; it can be seen that the film thickness is between 400 and $500 \mathrm{~nm}$, in agreement with previous results [7]. Comparable thickness (within experimental error) was registered for the other coatings.

CVD deposition is a quite complex process, in which many different parameters can affect the characteristics of the coatings; considering in particular AACVD, the presence of a solvent and the aerosol dynamics are additional parameters to be considered [35]. Surely the nature of the precursor can play a crucial role in determining the morphology, the phase formed and the most suitable deposition temperature
$[16,20,21]$; indeed, according to the molecular structure of the precursor and its stability, different growth mechanisms may occur. This can be caused by the formation of different gaseous intermediates during the deposition. This, in turn, can have an effect on the grains' formation on the surface of the substrate. The presence of other molecules and/or additives in the precursor solution can also affect the reactions taking place, influencing the growth rate and mechanisms and, consequently, the morphology [36].

Considering these factors, it was reasonable to expect some changes in the structure of the films when a modified precursor was employed; 
our results, therefore, confirm this. It has to be highlighted, however, that the addition of acac to the deposition solution, even at the lowest concentration, was the element leading to the most significant change in morphology. In fact, there are many more similarities in the structures of samples AA_0.5, AA_1 and AA_4 than with AA_0, i.e. the simple presence of acac was more determinant in the creation of the nanoparticles than the concentration itself. This would indicate that the acac ligand, even in small quantities (less than stoichiometric), has a remarkable effect on the deposition mechanisms - and consequently on the coatings' morphology (and composition, as shown by Raman data). Indeed, literature reports that in AACVD depositions the presence of organic molecules (i.e. surfactants) in the precursor solution led to significant difference in the morphology of the films [37,38]. The effect due to different acac concentrations, on the other hand, although visible, is less significant, as all three coatings AA_0.5, AA_1 and AA_4 show the same features (i.e. rounded crystallites), even if of different dimensions or distributions.

Fig. 4 shows, as an example, AFM micrographs of AA_0 and AA_4 (Fig. 4(a) and 4(b), respectively). AFM data confirm the differences in the morphologies of the two coatings on the micron scale. For the sample deposited without acac, very large grains can be observed (up to micron size in diameter); they are formed by the aggregation of smaller grains (hundreds of nanometers), and show a height of up to $150 \mathrm{~nm}$ on the z-axis. Images taken in different areas of the coating showed the same features. For sample AA_4, on the other hand, much smaller and more regular grains can be observed, and these have a maximum height of only $40 \mathrm{~nm}$ on the z-axis. The average roughness is 14.0 and $6.0 \mathrm{~nm}$ for coatings AA_0 and AA_4 respectively. Other samples prepared with different acac content presented similar features (data not shown).

Fig. 5(a) shows an example XPS spectrum of sample AA_4 for the titanium energy region (spectra for samples AA_0.5 and AA_1 showed similar features). The peak corresponding to Ti $2 \mathrm{p} 3 / 2$ can be detected, in the energy region around $455-460 \mathrm{eV}$. The most intense peak is detected at about $458.7 \mathrm{eV}$, belonging to $\mathrm{Ti}^{4+}$; this is in agreement with literature [39]. A smaller peak can be detected at $464.4 \mathrm{eV}$, belonging to Ti 2p1/2, again in agreement with literature. Previous analysis of AACVD $\mathrm{TiO}_{2}$ coatings prepared using TIPP as precursor showed the presence of $\mathrm{Ti}^{4+}$ only, with no further oxidation states observed $[25,40]$. Samples studied here deposited using acac, however, show the presence of different titanium environments; indeed, a shoulder in the main titanium peak can also be observed. With a mathematical deconvolution of this peak, as has been previously reported in literature [18], a signal belonging to $\mathrm{Ti}^{3+}$ can be detected, at $457.2 \mathrm{eV}$. Literature data report $\mathrm{Ti}^{3+}$ already being detected in $\mathrm{TiO}_{2}$ deposits [39,40], which is probably be due to the presence of oxygen vacancies.

The region corresponding to carbon $\mathrm{C} 1 \mathrm{~s}$ is shown in Fig. 5(b). It can be seen that a broad peak in the region $280-290 \mathrm{eV}$ is present, and the signal can be deconvoluted into different peaks, the most intense being at $284.8 \mathrm{eV}$ and corresponding to the $\mathrm{C}-\mathrm{C}$ bond in aliphatic carbon [41]. Weaker signals were detected at 286.4, 287.8 and $289.0 \mathrm{eV}$; they can be ascribed to $\mathrm{C}-\mathrm{O}, \mathrm{C}-\mathrm{O}$ and $\mathrm{O}-\mathrm{C}=\mathrm{O}$, respectively [42]. The spectra of the other samples prepared used acac showed a similar behaviour, i.e. carbon contamination present in the coatings. Although peaks belonging to carbon were previously detected in $\mathrm{TiO}_{2}$ coatings, it is worth highlighting that they were not present in deposits prepared by AACVD when just TIPP and no acac was employed as the precursor $[25,40]$.

Indeed, in these previous studies carbon concentration was below the XPS detection limit. This difference can be explained considering the lower reactivity / higher stability of the complex formed between $\mathrm{Ti}^{4+}$ and the acac ligand; this means that, in comparison with the simple TIPP molecule, more energy would be required for the complete degradation of the Ti-acac system. This, in turn, leads to the presence of some residual carbon in the coatings. These XPS results are in agreement with Raman analysis (see Fig. 2), which showed graphite-like carbon contamination when acac was used.
Using these spectra, a C/Ti ratio was calculated; all three samples prepared using acac showed a value of about $1 \mathrm{~mol} / \mathrm{mol}$; no significant difference was observed with different quantities of acac ligands bound to the titanium. This indicates that the stability of the Ti-acac complex does not change considerably according to the number of acac ligands.

Such presence of carbon in the coatings could also explain their amorphous nature; indeed, literature reports that carbon contamination can lead to lower crystallinity levels, as observed not only for titanium dioxide coatings, but also for other oxides [37,43]. In this case, as shown by XRD pattern in Fig. SI2, the material deposited without acac was already poorly crystalline; acac addition resulted in coatings which are completely amorphous.

The energy range corresponding to the O1s region is shown in Fig. 5(c)-(e) for samples AA_0.5, AA_1 and AA_4, respectively; all three samples are shown since the spectra present different features. In all three coatings, the peak can be deconvoluted into three signals, at about 530,532 and $534 \mathrm{eV}$; they correspond to the oxygen in $\mathrm{Ti}-\mathrm{O}$, in Ti-O-H bonds and residual water/organics oxygen, respectively $[16,44]$. While the water/organic peak is the less intense for all three samples, differences can be seen in the relative intensities of the other two signals, according to the different acac content. For AA_0.5, in fact, the most intense signal is the $\mathrm{Ti}-\mathrm{O}-\mathrm{H}$ one, while the $\mathrm{Ti}-\mathrm{O}$ is just a shoulder. For higher acac content, on the other hand, the Ti-O peak is the dominant one; for AA_4, in particular, Ti-O-H peak is just a shoulder.

This latter situation is the most similar to that previously observed in AACVD $\mathrm{TiO}_{2}$ coatings prepared with only TIPP as the precursor $[16,25]$. These different oxygen environments indicate different interactions of the surface oxygen with the possible atmospheric moisture, which can result in higher or lower $\mathrm{O}-\mathrm{H}$ surface content.

The optical behaviour of some samples is shown in Fig. 6, where the reflectance of the coatings is reported as a function of the wavelength (see Fig. SI4 for the data plotted in absorbance mode). When no acac is employed (AA_0), the profile is characteristic of $\mathrm{TiO}_{2}$, with a sharp increase in the reflectance at about $370 \mathrm{~nm}$, in the UV region. Adding acac to the deposition precursor leads to changes in the optical properties - in fact, for AA_1, a less-sharp increase in the reflectance was registered. For higher acac content (AA_4), the increase in the reflectance is shifted to higher wavelengths, in the visible region (about $450 \mathrm{~nm}$ ). This behaviour was previously reported for carbon-containing $\mathrm{TiO}_{2}$ samples [45]. The lower reflectance of the coatings deposited using acac can be explained considering the higher carbon content, hence darker colour; similar results were reported for other $\mathrm{TiO}_{2}$ coatings which contained carbon [46,47].

\subsection{Photocatalytic properties}

To test the photocatalytic activity under UVA light, resazurin degradation was monitored over time. Fig. SI5 shows, as an example, the curves for different times for AA_1. From these curves, FQE and FQY were calculated - Fig. 7(a) and (b), respectively.

Considering the FQE (Fig. 6(a)), it can be seen that samples prepared with acac added to the solution have better performance than the corresponding one with no ligand. The coating prepared with the smallest amount of acac (AA_0.5) shows a $30 \%$ improvement; for an acac:Ti ratio equal to 1 (AA_1), the greatest increase in performance is observed - indeed, the FQE value is three times higher. Increasing acac concentration even more (AA_4), the efficiency observed is about twice as high as for the unmodified sample. Such greater activity could be due to the presence of both anatase and rutile phases; as stated in the introduction, this can improve the photocatalytic efficiency. The presence of carbon in the coatings can also have an effect; literature indeed reports about increased photocatalytic activity for C-containing $\mathrm{TiO}_{2}$ coatings, as carbon can help reduce charge recombination $[48,49]$. Oxygen vacancies, detected in the coatings prepared with acac, can also enhance the photocatalytic efficiency [43]. Comparing the three films, 

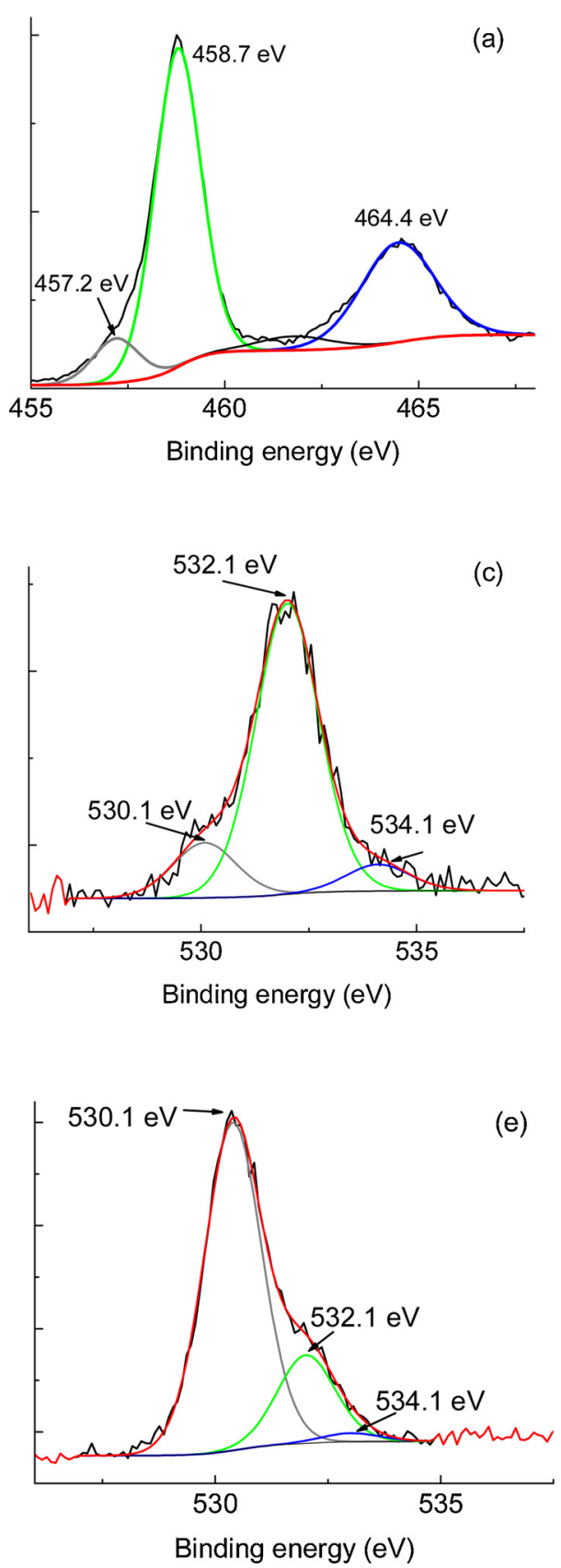
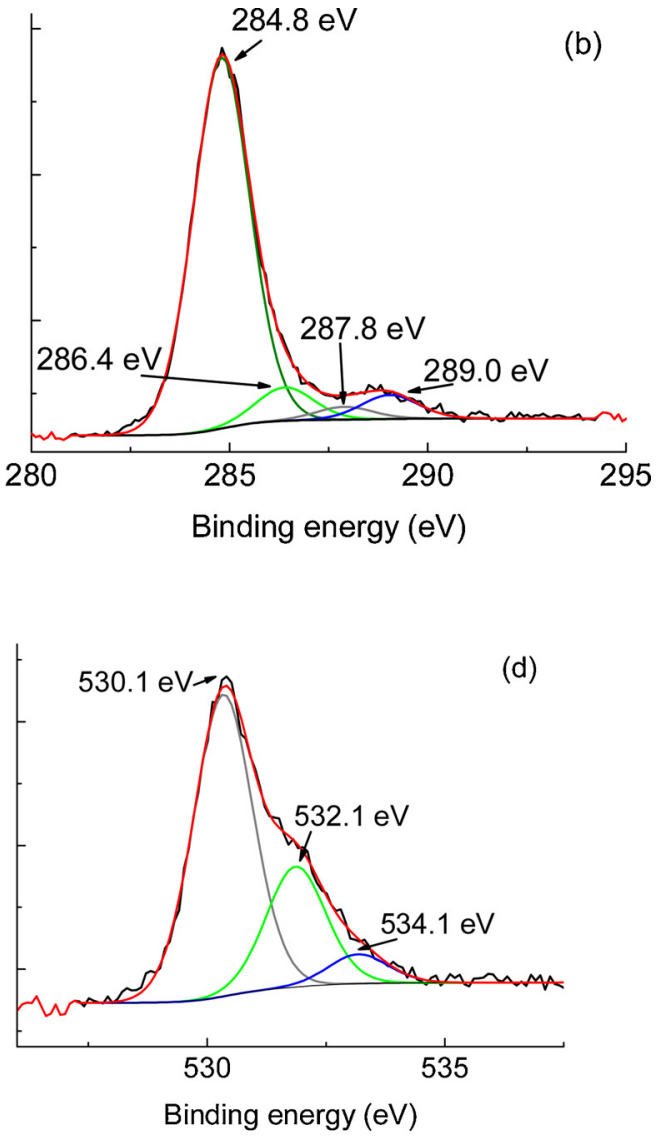

Fig. 5. XPS data for (a) Ti2p for sample AA_4; (b) C1s for sample AA_4; O1 s for (c) sample AA_0.5, (d) sample AA_1, (e) AA_4.

the presence of different quantities of $\mathrm{OH}$ group on their surface may also play a role in the different $\mathrm{FQE}$ values; this is an important point which should be investigated further.

Considering the FQY data (Fig. 7(b)), however, the effects of acac addition are less linear. If the number of photons absorbed is taken into account, in fact, samples AA_0.5 and AA_4 do not show better performance than AA_0; their FQY are, respectively, $30 \%$ lower and comparable to AA_0. Sample AA_1 shows the highest value, in agreement with FQE data; for samples AA_0.5 and AA_4, however, there was no improvement in the performance. In fact, their FQY values were smaller and about equal to that of sample AA_0, respectively.

The difference between FQE and FQY can be explained considering the different photon absorption by the various films - the values are reported in Table SI1. It can be seen, in fact, that the absorbed photon values for the coatings prepared with acac are more than double that for
AA_0 - $4.2 \times 10^{+12}$ vs. (9.4-9.5) $\times 10^{+12}$. These data indicate that coatings prepared using acac can absorb a higher number of photons, which is not significantly affected by the amount of acac employed. Literature reports that the morphology of the material can have an effect on photon absorption [50,51]; it has to be noted, however, that this is a complex process, with many parameters playing a role in it. The concentration of ligands has an effect on the photons which can actually generate the charged reactive species involved in the photocatalysis. Considering the resazurin degradation rate, the proportion is higher for sample AA_1 than for AA_0.5 and AA_4.

Considering this, it can be stated that the ratio of $1: 1$ between titanium and acac is the most suitable for AACVD deposition experiments, as it leads to the coating with the highest photocatalytic efficiency and yield. 


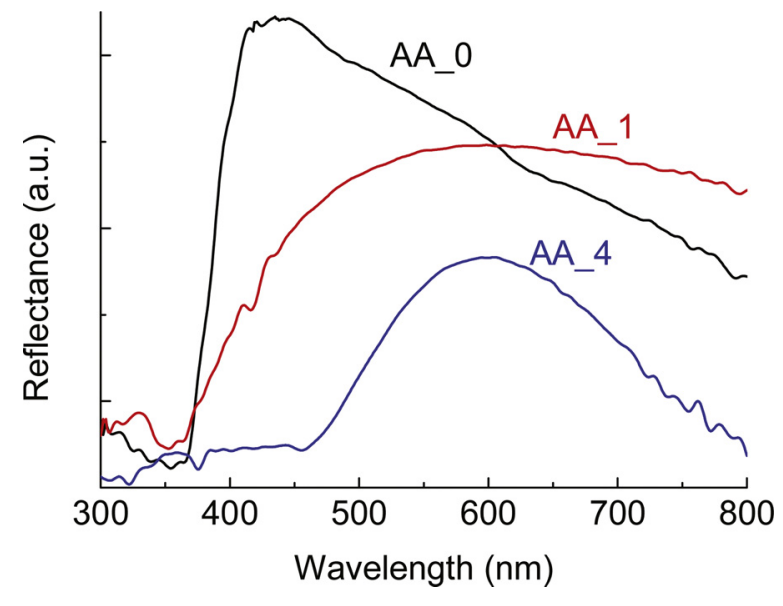

Fig. 6. UV-vis spectra for selected coatings.
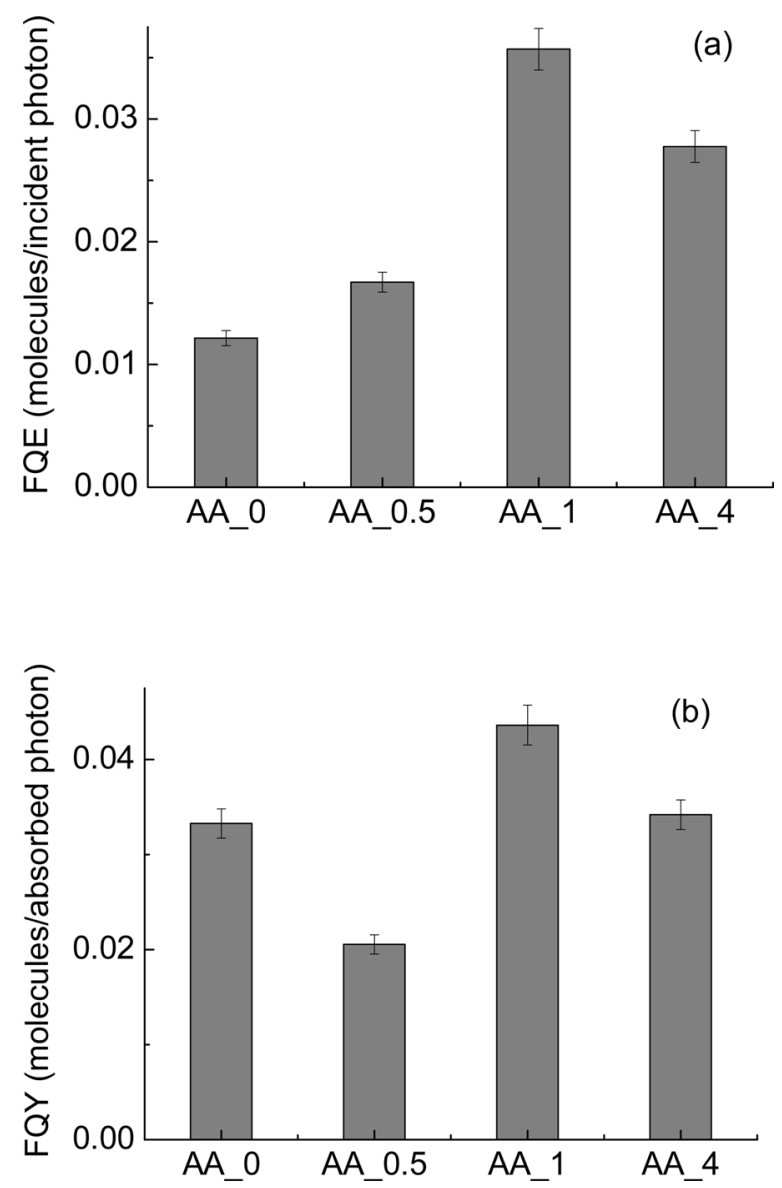

Fig. 7. (a) Formal Quantum Efficiency (FQE) and (b) Formal Quantum Yield (FQY) for the coatings deposited with various amounts of acac. The error was estimated to be about $5 \%$ (from previous work with the same apparaturs).

\section{Conclusions}

Titanium dioxide coatings were successfully deposited by AACVD technique using complexes between titanium and acac as precursors. Results showed that, employing acac, deposits with nanostructured morphology were obtained, and SEM images showed rounded nanoparticles of between 10-25 nm diameter. XRD measurements showed that deposits are amorphous; Raman analysis, on the other hand, indicated that both the anatase and rutile phases were present in the films. Raman and XPS also showed that a significant amount of residual carbon was incorporated into the titania coatings with acac; yet despite this, they also displayed photocatalytic properties similar to, or superior to, AACVD films produced without acac, which contained no carbon.

A ratio between titanium and acac equal to one led to the material with the highest photocatalytic activity.

Overall, these complexes were shown to be suitable precursors for $\mathrm{TiO}_{2}$ AACVD deposition. As future work, post-deposition treatments (i.e. annealing) of the coatings may be considered, to partially reduce the carbon contamination evidenced by XPS analysis and see the possible correlation on the photoactivity. Moreover, possible differences in hydrophilic/hydrophobic character of the coatings, due to the different surface $\mathrm{OH}$, should be further investigated.

\section{Authors statement}

Clara Piccirillo and Ivan Parkin designed the experiments, Megan Taylor and Clara Piccirillo performed the CVD depositions and measured the photocatalytic activity, Robert Pullar performed the characterisation analysis. All authors were involved in the discussion of the data and writing of the paper.

\section{Declaration of Competing Interest}

On behalf of all authors, I declare that we have no conflict of interest.

\section{Acknowledgments}

Clara Piccirillo would like to thank Fondazione con il Sud, for funding the project HApECOrk (2015-0243). Robert C. Pullar thanks the FCT (Fundação para a Ciência e Tecnologia, Portugal) for supporting this work through grant IF/00681/2015. This work was developed within the scope of the project CICECO - Aveiro Institute of Materials, POCI-01-0145-FEDER-007679 FCT Ref. UIDP/50011/2020, financed by national fundsand when appropriate co-financed by FEDER under the PT2020 Partnership Agreement through the FCT/MCTES. Authors would like to thank Dr. David Tobaldi for the help with the interpretation of UV spectroscopy data and Dr. Sonia Carallo for the AFM measurements.

\section{Appendix A. Supplementary data}

Supplementary material related to this article can be found, in the online version, at doi:https://doi.org/10.1016/j.jphotochem.2020. 112727.

\section{References}

[1] V. Rodríguez-González, C. Terashima, A. Fujishima, Application of photocatalytic titanium dioxide-based nanomaterials in sustainable agriculture, J. Photochem. Photobiol. C Photochem. Rev. 40 (2019) 49-67.

[2] A. Yamakata, J.J.M. Vequizo, Curious behaviour of photogenerated electrons and holes at the defects on anatase, rutile, and brookite TiO2 powders: a review, J. Photochem. Photobiol. C Photochem. Rev. 40 (2019) 234-243.

[3] D.M. Tobaldi, R.C. Pullar, M.P. Seabra, J.A. Labrincha, Fully quantitative X-ray characterisation of evonik aeroxide TiO2 P25® Mater. Lett. 122 (2014) 345-347.

[4] D.M. Tobaldi, C. Piccirillo, N. Rozman, R.C. Pullar, M.P. Seabra, A. Sever Škapin, P.M.L. Castro, J.A. Labrincha, Effects of $\mathrm{Cu}, \mathrm{Zn}$ and $\mathrm{Cu}-\mathrm{Zn}$ addition on the microstructure and antibacterial and photocatalytic properties of Cu-Zn modified TiO2 nano-heterostructures, J. Photochem. Photobiol. A: Chem. 330 (2016) 44-54.

[5] K. Page, R.G. Palgrave, I.P. Parkin, M. Wilson, S.L.P. Savin, A.V. Chadwick, Titania and silver-titania composite films on glass - potent antimicrobial coatings, J. Mater. Chem. 17 (2007) 95-104.

[6] R. Qian, H. Zong, J. Schneider, G. Zhou, T. Zhao, Y. Li, J. Yang, D.W. Bahemann, J.H. Pan, Charge carrier trapping, recombination and transfer during TiO2 photocatalysis: an overview, Catal. Today 335 (2019) 78-90.

[7] L. Romero, C. Piccirillo, P.M.L. Castro, C. Bowman, M.E.A. Warwick, R. Binions, Titanium dioxide thin films deposited by electric field-assisted CVD: effect on antimicorbial and photocatalytic properties, Chem. Vap. Depos. 21 (2015) 63-70.

[8] A. Anderson, R. Binions, A preferential precursor for photocatalytically active titanium dioxide thin films: titanium bis-ammonium lactate dihydroxide as an 
alternative to titanium tetra iso-propoxide, Polyhedron 118 (2016) 81-90.

[9] M. Krunks, I. Oja, K. Tõnsaadu, M. Es-Souni, M. Gruselle, L. Niinistö, Thermoanalytical study of acetylacetonate-modified titanium(IV) isopropoxide as a prepcursor for TiO2 films, J. Therm. Anal. Calor. 80 (2005) 483-488.

[10] K. Farhadian Azizi, M.M. Bagheri-Mohagheghi, Transition from anatase to rutile phase in titanium dioxide (TiO2) nanoparticles synthesized by complexing sol-gel process: effect of kind of complexing agent and calcinating temperature, J. Sol-Gel Sci. Technol. 65 (2013) 329-335.

[11] I. Oja Acik, N.G. Oyekoya, A. Mere, A. Loot, L. Dolgov, V. Mikli, M. Krunks, I. Sil, Plasmonic TiO2:Au composite layers deposited in situ by chemical spray pyrolysis, Surf. Coat. Technol. 271 (2015) 27-31.

[12] S. John, A. Salam, A.M. Baby, A. Joseph, Corrosion inhibition of mild steel using chitosan / TiO2 nanocomposite coatings, Prog. Org. Coat. 129 (2019) 254-259.

[13] K. Usha, P. Kumbhakar, B. Mondal, Effect of Ag-doped TiO2 thin film passive layers on the performance of photo-anodes for dye-sensitized solar cells, Mater. Sci. Semicond. Process. 43 (2016) 17-24.

[14] S. Suárez, N. Arconado, Y. Castro, J.M. Coronado, R. Portela, A. Durán, B. Sánchez, Photocatalytic degradation of TCE in dry and wet air conditions with TiO2 porous thin films, Appl. Catal. B 108 (2011) 14-21.

[15] I. Stambolova, M. Shipochka, V. Blaskov, A. Loukanov, S. Vassilev, Sprayed nanostructured TiO2 films for efficient photocatalytic degradation of azo textile azo dye, J. Photochem. Photobiol. B 117 (2012) 19-26.

[16] B.J. Blackburn, C. Drosos, D.B. Brett, M.A. Parkes, C.J. Carmalt, I.P. Parkin, In situ mass spectrometry analysis of chemical vapour deposition of $\mathrm{TiO} 2$ thin films to study gas phase mechanisms, RSC Adv. 6 (2016) 111797-111805.

[17] A. Perraudeau, C. Dublanche-Tixier, P. Tristant, C. Chazelas, Dynamic mode optimization for the deposition of homogeneous TiO2 thin film by atmospheric pressure PECVD using microwave plasma torch, Appl. Surf. Sci. 493 (2019) 703-709.

[18] A. Lepcha, et al., Electrospun black titania naofibers: influence of hydrogen plasmainduced disorder on the electronic structure and photoelectrochemical performance, J. Phys. Chem. C 119 (2015) 18835-18842.

[19] C. Fàbrega, D. Monllor-Satoca, S. Ampudia, A. Parra, T. Andreu, J.R. Morante, Tuning the Fermi level and the kinetics of surface states of TiO2 nanorods by means of ammonia treatments, J. Phys. Chem. C 117 (2013) 20517-20524.

[20] S.J. Kim, et al., Intrinsic nitrogen-doped CVD-grown TiO2 thin films from All-Ncoordinated Ti precursors for photoelectrochemical applications, Chem. Vap. Depos. 19 (2013) 45-52.

[21] C. Edusi, G. Sankar, I.P. Parkin, The effect of solvent on the phase of titanium dioxide deposited by aerosol-assisted CVD, Chem. Vap. Depos. 18 (2012) 126-132.

[22] A.J. Gardecka, G.K.L. Goh, G. Sankar, I.P. Parkin, On the nature of niobium substitution in niobium doped titania thin films by AACVD and its impact on electrical and optical properties, J. Mater. Chem. A 3 (2015) 17755-177762.

[23] S.P. Lim, N.M. Huang, H.N. Lim, M. Mazhar, Aerosol assisted chemical vapour deposited (AACVD) of TiO2 thin film as compact layer for dye-sensitised solar cells, Ceram. Int. 40 (2014) 8045-8052.

[24] C. Edusi, G. Hyett, G. Sankar, I.P. Parkin, Aerosol-assisted CVD of titanium dioxide thin films from methanolic solution of titanium tetraisopropoxide; substrate and aerosol-selective deposition of rutile or anatase, Chem. Vap. Depos. 17 (2011) 30-36.

[25] C. Piccirillo, C.J. Denis, R.C. Pullar, R. Binions, I.P. Parkin, J.A. Darr, P.M.L. Castro, Aerosol assisted chemical vapour deposition of hydroxyapatite-embedded titanium composite thin films, J. Photochem. Photobiol. A 332 (2017) 45-52.

[26] A.M. Aloitabi, S. Sathasivam, I.P. Parkin, Aerosol assisted chemical vapour deposition of a $\mathrm{ZrO} 2-\mathrm{TiO} 2$ composite thin film with enhanced photocatalytic activity, RSC Adv. 5 (2015) 67944

[27] S. Javed, M. Mujahid, M. Islam, M.A. Akram, Precursor modification and refluxing effects on titania nanostructures prepared via sol gel reflux synthesis, TMS 2013 (2013) 127-134.

[28] P. Garra, F. Morlet-Savary, B. Graff, F. Dumur, V. Monnier, C. Dietlin, D. Gigmesh, J.P. Fouassiera, J. Lalevée, Metal acetylacetonate-bidentate ligand interaction (MBLI) as highly efficient free radical generating system for polymer synthesis, Polym. Chem. 9 (2018) 1371-1378.

[29] H. Assi, G. Mouchaham, N. Stenou, T. Devic, C. Serre, Titanium coordination compounds: from discrete metal complexes to metal-organic frameworks, Chem. Soc. Rev. 46 (2017) 3431-3452.

[30] F. Caruso, C. Pettinari, F. Marchetti, P. Natanti, C. Phillips, J. Tanski, M. Ross, Synthesis, molecular structure (X-ray and DFT), and solution behavior of titanium 4-acyl-5-pyrazolonates. Correlations with related antitumor $\beta$-diketonato derivatives, Inorg. Chem. 46 (2007) 7553-7560.

[31] R.M. Lord, J.J. Mannion, B.D. Crossley, A.J. Hebden, M.W. McMullon, J. Fisher, R.M. Phillips, P.C. McGowan, $\beta$-diketonate titanium compounds exhibiting high in vitro activity and specific dna base binding, Chem. Sel. 1 (2016) 6598-6605.

[32] P.N. Kumar Reddy, D.P.M.D. Shaik, V. Ganesh, D. Nagamalleswari, K. Thyagarajan, P. Vishnu Prasanth, Structural, optical and electrochemical properties of TiO2 nanoparticles synthesized using medicinal plant leaf extract, Ceram. Int. 45 (2019) 16251-16260.

[33] P. Periyat, D.E. McCormack, S.J. Hinder, S.C. Pillai, One-pot synthesis of anionic (nitrogen) and cationic (sulphur) codoped high-temperature stable, visible light active, anatase photocatalysts, J. Phys Chem. C 113 (2009) 3246-3253.

[34] R. Liu, H. Li, L. Duan, H. Shen, Q. Zhang, X. Zhao, Influences of annealing atmosphere on phase transition temperature, optical properties and photocatalytical activites of TiO2 phase junction microspheres, J. Alloys Comp. 789 (2019) 1015-1021.

[35] T.S. Chadha, M. Yang, K. Haddad, V.B. Shah, S. Li, P. Biswas, Model based prediction of nanostructured thin film morphology in an aerosol chemical vapour deposition process, Chem. Eng. J. 310 (2017) 102-113.

[36] S. Chen, R.M. Wilson, R. Binions, Synthesis of highly surface-textured ZnO thin films by aerosol assisted chemical vapour deposition, J. Mater. Chem. A 3 (2015) 5794-5797.

[37] M. Saeli, R. Binions, C. Piccirillo, I.P. Parkin, Templated growth of smart coatings: hydrid chemical vapour deposition of vanadyl acetylacetonate with tetraoctyl ammonium bromide, Appl. Surf. Sci. 225 (2009) 7291-7295.

[38] R.A. Hussain, A. Badshah, A. Younis, M.D. Khan, J. Akhtar, Iron selenide films by aerosol assisted chemical capor deposition from single source organometallic precursor in the presence of surfactants, Thin Sol. Films 567 (2014) 58-63.

[39] S. Naseem, I.V. Pinchuk, Y.K. Luo, R.K. Kawakami, S. Khan, S. Husain, W. Khan, Epitaxial growth of cobalt doped TiO2 thin films on LaAlO3 (100) substrate by molecular beam epitaxy and their opto-magnetic based applications, Appl. Surf. Sci. 493 (2019) 691-702.

[40] D.S. Bhachu, et al., Solution processing route to multifunctional titania thin films: highly conductive and photocatalytically active Nb:TiO2, Adv. Funct. Mater. 24 (2014) 5075-5085.

[41] S. Franchi, V. Secchi, M. Santi, M. Dettin, A. Zamuner, C. Battocchio, G. Iucci, Biofunctionalization of TiO2 surfaces with self-assembling oligopeptides in different $\mathrm{pH}$ and ionic strength conditions: charge effects and molecular organization, Mater. Sci. Eng. C 90 (2018) 651-656.

[42] P.M. Jayaweera, E.L. Quah, H. Idriss, Photoreaction of ethanol on TiO2 (110) single-crystal surface, J. Phys. Chem. C 111 (2007) 1764-1769.

[43] A.V. Raghu, K.K. Karuppanan, B. Pullithadathil, Controlled carbon doping in anatase TiO2 (101) facets: superior trace-level ethanol gas sensor performance and adsorption kinetics, Adv. Mater. Interface 6 (2019) 1801714.

[44] M.K. Lee, Y.C. Park, Contact angle relaxation and long-lasting hydrophilicity of sputtered anatase TiO thin films by novel quantitative XPS analysis, Langmuir 35 (2019) 2066-2077.

[45] Z. Noorimotlagh, I. Kazeminezhad, N. Jaarfarzadeh, M. Ahmadi, Z. Ramezani, Improved performance of immobilized TiO2 under visible light for the commercial surfactant degradation: role of carbon doped $\mathrm{TiO} 2$ and anatase/rutile ratio, Catal. Today 348 (2020) 277-289.

[46] L.V. Mohan, S.M. Shiva Nagendra, M.P. Maiya, PPhotocatalytic degradation of gaseous toluene using slef-assembled air filter based on chitosan/activated carbon/ TiO2, J. Env. Chem. Eng. 7 (2019) 103455.

[47] A. Ansón-Casaos, M.J. Sampaio, C. Jarauta-Córdoba, M.T. Martínez, C.G. Silva, J.L. Faria, A.M.T. Silva, Evaluation of sol-gel TiO2 photocatalysts modified with carbon or boron compounds and crystallyzed in nitrogen or air atmospheres, Chem. Eng. J. 277 (2015) 11-20.

[48] F. Liu, N. Feng, L. Yang, Q. Wang, J. Xu, F. Deng, Enhanced photocatalytic performance of carbon-coated TiO2-x with surface-active carbon species, J. Phys. Chem. C 122 (2018) 10948-10955.

[49] M. Wang, J. Han, G. Yuan, R. Guo, Carbon/TiO2/Fe2O3 hybrid shells as efficient visible light photocatalyst, New J. Chem. 43 (2019) 11282-11287.

[50] A. Das, S.K. Nikhil, R.G. Nai, Influence of surface morphology on photocatalytic performance of zinc oxide: a review, Nano-Struct. Nano-Objects 19 (2019) 100353

[51] B.Y. Yu, S.Y. Kwak, Carbon quantum dots embedded with mesoporous hematite nanospheres as efficient visible light-active photocatalysts, J. Mater. Chem. 22 (2012) 8345-8353. 